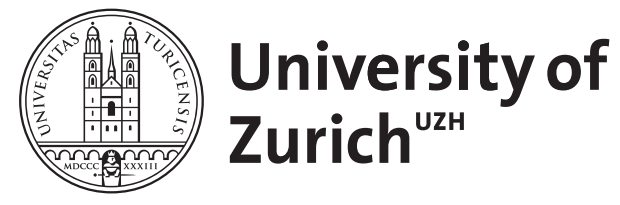

\title{
Spinal phenotype of cerebrotendinous xanthomatosis
}

\author{
Bartholdi, D ; Zumsteg, D ; Verrips, A ; Wevers, R A ; Sistermans, E ; Hess, K ; Jung, H H
}

DOI: https://doi.org/10.1007/s00415-004-0221-x

Posted at the Zurich Open Repository and Archive, University of Zurich

ZORA URL: https://doi.org/10.5167/uzh-155780

Journal Article

Published Version

Originally published at:

Bartholdi, D; Zumsteg, D; Verrips, A; Wevers, R A; Sistermans, E; Hess, K; Jung, H H (2004). Spinal phenotype of cerebrotendinous xanthomatosis. Journal of Neurology, 251(1):105-107.

DOI: https://doi.org/10.1007/s00415-004-0221-x 

D. Bartholdi
D. Zumsteg
A. Verrips
R. A. Wevers
E. Sistermans
K. Hess
H. H. Jung

\section{Spinal phenotype of cerebrotendinous xanthomatosis \\ A pitfall in the diagnosis of multiple sclerosis}

Received: 17 April 2003

Received in revised form: 24 June 2003

Accepted: 4 July 2003

Sirs: Cerebrotendinous xanthomatosis (CTX; MIM\#213700) is an autosomal recessive lipid storage disorder caused by deficiency of the enzyme sterol-27-hydroxylase (CYP27) [1-3]. CYP27 mutations impair the primary bile acids synthesis, leading to accumulation of cholesterol and cholestanol in various tissues, particularly in the central and peripheral nervous systems, eye lenses, and tendons [2]. The "classical" CTX phenotype includes the triad of juvenile cataracts, tendon xanthomas, in particular of ankle tendons, and progressive nervous system involvement [4-6].

Neurological manifestations include cognitive decline, psychiatric disorders, epileptic seizures, pyramidal tract dysfunction, cerebellar signs, and a sensory-motor neuropathy characterized by reduced numbers of large myelinated axons. Disease onset varies from early infancy to middle adulthood [6]. Neuroradiological characteristics include cerebral and cerebellar white matter hyperintensities, and distinct signal alterations of the cerebellar dentate nuclei [7]. CTX may be successfully treated with chenodeoxycholic acid [8].
Herein, we report a non-consanguineous Swiss CTX family with a compound heterozygous mutation in the CYP27 gene, displaying a striking intra-familial phenotypic variability. While one patient presented with "classical" clinical signs of CTX, her older sister had a slowly progressive myelopathy as the only manifestation of the disease.

At the time of the evaluation, the index patient (patient 1) was 51 years old. She had a normal childhood psychomotor development, an average school performance, and a professional training as office employee. Since early childhood she had chronic diarrhea without identifiable cause in spite of repeated gastro-intestinal tract investigations. Aged 25 years, gait disturbance was noted for the first time. Since the symptoms manifested after a banal viral infection, a parainfectious meningo-myelitis was suspected. However, gait disturbance gradually progressed over the following years. Several cerebro-spinal fluid (CSF) examinations were normal. At the age of 35 years, the patient underwent bilateral cataract operation. Aged 45 years, behavioral abnormalities and cognitive decline became evident. Four years later, she had a first episode of severe depression. Cerebral magnetic resonance imaging (MRI) was reported to be normal. At the age of 51 years, the patient was admitted to our department for further evaluation. General physical examination showed no skin lesions, in particular no tendon xanthomas. Cognitive testing revealed severe impairment of short term memory and abstract reasoning. Neurological examination revealed a severely spastic-ataxic gait, increased muscle tone, mild leg weakness, brisk tendon reflexes with bilateral ankle clonus, extensor plantar responses, and reduced vibration and position sense of the feet. T2- and FLAIRweighted cerebral MRI revealed areas of increased signal intensity in the periventricular white matter and the cerebellum, including the dentate nuclei (Fig.).

Her one-year-older sister ( $p a-$ tient 2) had also a normal childhood psychomotor development, a good school performance, and underwent a professional training as secretary. After the age of 25 years, she developed progressive gait disturbance. Although repeated CSF analysis including oligoclonal focusing, and visually evoked potentials were normal, diagnosis of a primary progressive multiple sclerosis was made. At age 28 years, oral methotrexate treatment was started but discontinued after a few years because of adverse effects. Over the following years, gait disturbance was slowly progressive. Repeated cerebral MRI's between 1989 and 1998 were normal, and no other neurological or systemic signs or symptoms evolved. In particular, there were no diarrhea, cataracts, skin or tendon alterations, impaired cognitive functions, or psychiatric symptoms. At the age of 52 years, she presented at our department for further evaluation. There were no cataracts or tendon xanthomas. Neurological examination revealed a spasticataxic gait, increased muscle tone, brisk tendon reflexes, extensor plantar responses, and slightly impaired vibration and position sense of the feet. Further clinical neurological testing, including assessment of cognitive functions, was normal.

The combination of chronic diarrhea, juvenile-onset cataracts, cognitive impairment, psychiatric symptoms, progressive neurological signs, and leukoencephalopathy including the dentate nuclei in the index patient prompted us to carry out an evaluation for CTX. Analysis of serum cholestanol and chole- 

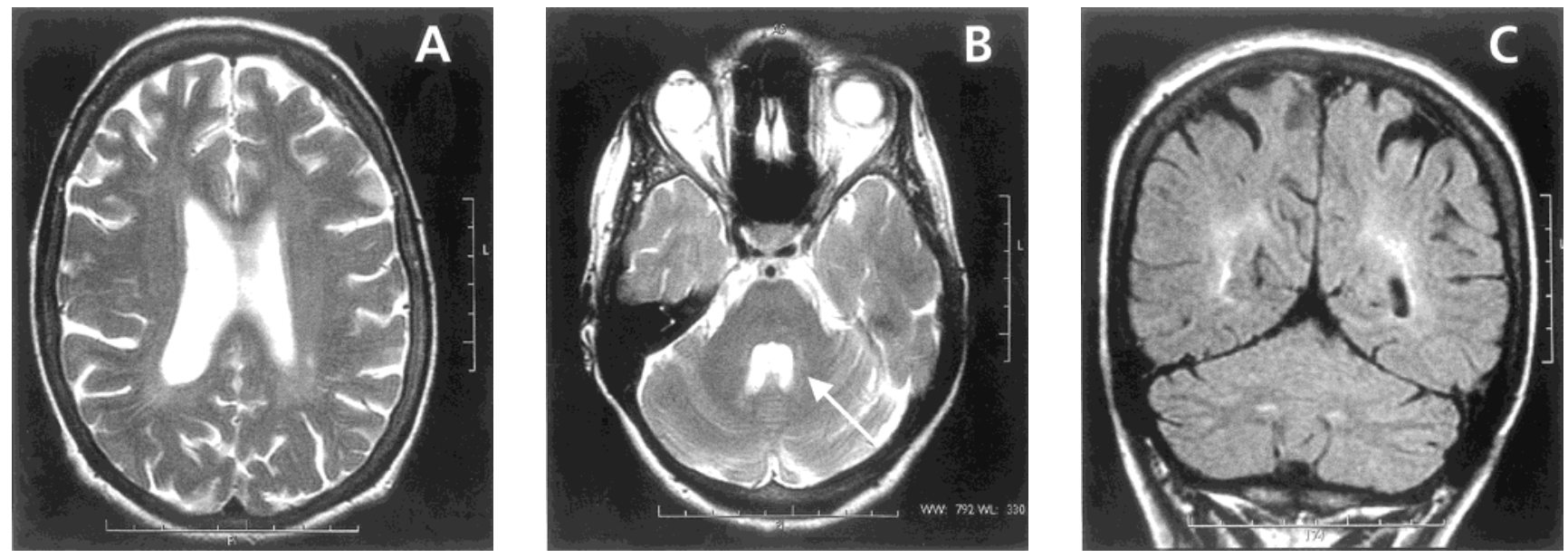

Fig. Cerebral Magnetic Resonance Imaging. Axial T2-weighted images of patient 1 demonstrated supratentorial signal abnormalities predominantly found in the posterior periventricular white matter (A). Additional infratentorial signal abnormalities were located in the dentate nuclei (arrow; B). The pathological findings are confirmed with coronar FLAIR images (C)

sterol levels, as well as urine bile alcohol excretion were performed as previously described [1]. Both sisters had highly increased serum cholestanol levels and urine bile alcohol excretion, thus confirming CTX diagnosis (Table). Sequence analysis of the CYP27 gene was carried out as previously described [6]. Both sisters were compound heterozygous for the missense mutations $646 \mathrm{G}>\mathrm{C}(\mathrm{A} 216 \mathrm{P})$ and $1213 \mathrm{C}>\mathrm{T}(\mathrm{R} 405 \mathrm{~W})$. The daughter of the index patient, being an obligate heterozygous mutation carrier, had normal biochemical findings (Table). Treatment with chenodeoxycholic acid was started in both sisters, and follow-up examinations are ongoing.

Originally, CTX was defined by the clinical trial of tendon xanthomas, bilateral juvenile-onset cataracts, and progressive neurological deterioration $[4,5]$. How- ever, further clinical observations demonstrated an extended spectrum of CTX phenotypes. Tendon xanthomas were found in only two thirds of patients with otherwise "classical" CTX $[6,11]$. In contrast, chronic intractable diarrhea was not noted initially, but was reported in more than a third of patients in recent series [11]. In addition, several patients had a predominantly spinal CTX phenotype [10]. This so-called "spinal xanthomatosis" was characterized by a slowly progressive myelopathy without cerebellar dysfunction, cognitive decline, or tendon xanthomas [10]. As in our patient, individuals with the spinal CTX phenotype were prone to be misdiagnosed as primary progressive multiple sclerosis [10].

Both mutations found in our patients had been previously described $[6,9]$. The $646 \mathrm{G}>\mathrm{C}$ muta-

Table Biochemical Findings

\begin{tabular}{lccl}
\hline & Patient 1 & Patient 2 & $\begin{array}{l}\text { Relative with heterozygous } \\
\text { CYP27 mutation }\end{array}$ \\
\hline Cholestanol Serum $(\mu \mathrm{mol} / / ; \mathrm{N}=3.3-12.5)$ & 139 & 69 & 7 \\
Cholesterol Serum $(\mathrm{mmol} / / \mathrm{N}=4.7-6.5)$ & 6.4 & 7.0 & 4.3 \\
Cholestanol/Cholesterol Ratio $(\% ; \mathrm{N}<0.21)$ & 2.17 & 0.98 & 0.16 \\
Bile Alcohols Urine $(0-+++; \mathrm{N}=0)$ & +++ & +++ & 0 \\
\hline
\end{tabular}

tion results in the replacement of an alanine residue by a proline at the last position of exon 3 . The mutation leads to very low levels of CYP27 mRNA, thus confirming its pathogenicity [9]. Most homozygous $646 \mathrm{G}>\mathrm{C}$ mutation carriers develop the severe "classical" CTX phenotype with early childhood onset [9]. The 1213C $>$ T mutation results in the replacement of an arginine residue by a tryptophane in exon 7. The mutation exchanges an evolutionary strongly conserved amino acid within the coding region for the adrenodoxin- and heme-binding sites, which are critical for the CYP27 enzyme activity. The 1213C $>$ T mutation has only been observed in compound heterozygotes so far $[6,10]$.

The index patient presented with several key signs of CTX, namely juvenile-onset cataracts and diarrhea. In contrast, her sister carrying the identical mutations had a "spinal xanthomatosis" without additional manifestations of the disease. Although supplementary CTX-related signs and symptoms might still develop in the future, the long disease course represents a strong argument for a striking intra-familial phenotypic variability. Noteworthy, twice as high serum cholestanol levels were 
found in the more severely affected index patient compared with her sister. Although no conclusions can be drawn from two patients, and controlled studies of biochemical markers and disease severity are lacking, our findings suggest a correlation between the extent of the bile acid metabolism impairment and the clinical phenotype. One probable explanation for the phenotypic variability comprises individual differences in additional metabolic pathways interfering with tissue accumulation of cholesterol and cholestanol, as the capability of macrophages to eliminate sterols. Since both patients are compound heterozygous, preferential activation of one allele resulting in a more severe phenotype than activation of the other allele has to be considered as an alternative explanation for the phenotypic difference.

In conclusion, our findings confirm that CTX may exhibit a striking intra-familiar phenotypic variability. In addition, our report emphasizes the importance of CTX in the differential diagnosis of multiple sclerosis. CTX is most probably an underdiagnosed entity, in particular in patients with a spinal CTX phenotype. We propose that all patients with suspected primary progressive multiple sclerosis should be interrogated for chronic diarrhea. A further biochemical screening for CTX should be considered in all patients with a progressive spinal cord syndrome without convincing clinical, laboratory, or neuroradiological support for multiple sclerosis, or another distinct cause of myelopathy. CTX screening is even mandatory if two of four clinical criteria are present: 1) juvenile-onset cataracts, 2) chronic intractable diarrhea, 3 ) tendon xanthomas, and 4) progressive neurological symptoms. Although CTX is a rare disorder, early diagnosis is crucial because neurological progression may be slowed or even prevented by an early appropriate treatment.

\section{References}

1. Cali JJ, Russell DW (1991) Characterization of human sterol 27-hydroxylase. A mitochondrial cytochrome P-450 that catalyzes multiple oxidation reaction in bile acid biosynthesis. J Biol Chem 266:7774-7778

2. Cali JJ, Hsieh CL, Francke U, Russell DW (1991) Mutations in the bile acid biosynthetic enzyme sterol 27-hydroxylase underlie cerebrotendinous xanthomatosis. J Biol Chem 266:7779-7783

3. Björkhem I, Boberg KM (1995) Inborn errors in bile acid biosynthesis and storage of sterols other than cholesterol. In: Scriver CR, Beaudet $\mathrm{AL}$, William SS, Valle D (eds) The metabolic and molecular bases of inherited disease. McGraw-Hill, New York, pp 2082-2088

4. van Bogaert L, Scherer HJ, Epstein E. Une forme cérébrale de la cholesterinose généralisé. 1937. Paris, Masson et Cie

5. Schimschock JR, Alvord EC, Jr, Swanson PD (1968) Cerebrotendinous xanthomatosis. Clinical and pathological studies. Arch Neurol 18:688-698

6. Verrips A, Hoefsloot LH, Steenbergen GC, Theelen JP, Wevers RA, Gabreels FJ, Van Engelen BG, van den Heuvel LP (2000) Clinical and molecular genetic characteristics of patients with cerebrotendinous xanthomatosis. Brain 123:908-919

7. De Stefano N, Dotti MT, Mortilla M, Federico A (2001) Magnetic resonance imaging and spectroscopic changes in brains of patients with cerebrotendinous xanthomatosis. Brain 124: 121-131
8. Berginer VM, Salen G, Shefer S (1984) Long-term treatment of cerebrotendinous xanthomatosis with chenodeoxycholic acid. N Engl J Med 311: 1649-1652

9. Garuti R, Lelli N, Barozzini M, Tiozzo R, Dotti MT, Federico A, Ottomano AM, Croce A, Bertolini S, Calandra S (1996) Cerebrotendinous xanthomatosis caused by two new mutations of the sterol-27-hydroxylase gene that disrupt mRNA splicing. J Lipid Res 37: 1459-1467

10. Verrips A, Nijeholt GJ, Barkhof F, Van Engelen BG, Wesseling P, Luyten JA, Wevers RA, Stam J, Wokke JH, van den Heuvel LP, Keyser A, Gabreels FJ (1999) Spinal xanthomatosis: a variant of cerebrotendinous xanthomatosis. Brain 122:1589-1595

11. Verrips A, Van Engelen BG, Wevers RA, van Geel BM, Cruysberg JR, van den Heuvel LP, Keyser A, Gabreels FJ (2000) Presence of diarrhea and absence of tendon xanthomas in patients with cerebrotendinous xanthomatosis. Arch Neurol 57:520-524

D. Bartholdi · D. Zumsteg · K. Hess ·

H. H. Jung, MD ( $\varangle)$

Dept. of Neurology

University Hospital Zürich

Frauenklinikstrasse 26

8091 Zürich, Switzerland

Tel.: + 41-1/255-5545

Fax: +41-1/255-4507

E-Mail: hans.jung@nos.usz.ch

D. Bartholdi

Institute of Medical Genetics

University of Zürich

Zürich, Switzerland

A. Verrips

Dept. of Pediatric Neurology

University Medical Center

Nijmegen, The Netherlands

R. A. Wevers

Laboratory of Pediatrics \& Neurology

University Medical Center

Nijmegen, The Netherlands

E. Sistermans

Dept. of Human Genetics

University Medical Center

Nijmegen, The Netherlands 